DOI:

10.1038/nrn2122

\title{
Weeding out dopaminergic neurons
}

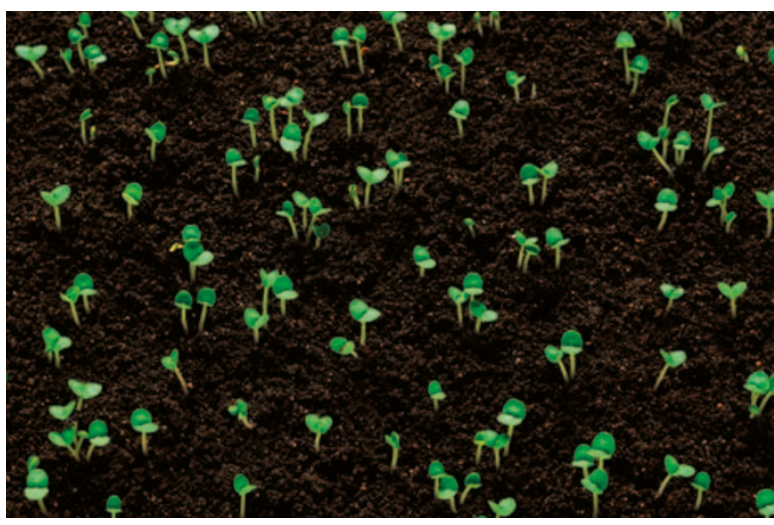

Parkinson's disease is a prime example of a disorder that has an environmental and a genetic component, but the precise role of these two factors in its aetiology is unknown. Chaudhuri and colleagues show that, in Drosophila, genes involved in dopamine synthesis and metabolism influence the susceptibility of dopaminergic neurons to oxidative damage caused by the herbicide paraquat.

Flies that had eaten paraquat had elevated activity of the enzyme catalase, an early indicator of paraquat-generated oxidative stress, in their heads. Paraquat ingestion also caused movement disorders that resembled those seen in people with Parkinson's disease. Giving L-3,4dihydroxyphenylalanine (L-DOPA), a dopamine precursor, or dopamine along with paraquat, almost completely prevented the motor deficits and increased the flies' life spans.

Since the effects of paraquat could be modified by manipulating dopamine levels, the researchers investigated whether paraquat induced the loss of dopaminergic neurons. They used transgenic flies in which expression of the green fluorescent protein $(G F P)$ gene was driven by the promoter of the gene for tyrosine hydroxylase $(\mathrm{TH})$, the enzyme that synthesizes dopamine from tyrosine. This allowed the researchers to visualize dopaminergic neurons, and they found that paraquat reduced the number of neurons in many dopaminergic neuron clusters. The herbicide did not kill cholinergic neurons or neurons in general, indicating that paraquat affects dopaminergic neurons specifically.

These findings suggested that paraquat alters dopamine neuron function, and the authors therefore set out to determine whether early changes in dopamine synthesis and metabolism might be measurable soon after paraquat exposure. Indeed, flies had reduced levels of dopamine and increased concentrations of L-DOPA and the dopamine metabolite 3,4-dihydroxyphenylacetic acid (DOPAC) 6 hours after paraquat ingestion.

Paraquat's capacity to alter dopamine synthesis and metabolism and kill dopaminergic neurons led the authors to investigate whether variations in genes that are involved in dopamine production and breakdown may confer sensitivity to paraquat-induced oxidative stress. They examined flies carrying heterozygous mutations in the genes pale (ple), which encodes TH; Punch (Pu), which encodes GTP cyclohydrolase 1 (GTPCH), an enzyme that is also involved in dopamine production; and Catecholamines-up (Catsup), the product of which inhibits dopamine synthesis. Heterozygous Catsup mutants were resistant to $20 \mathrm{mM}$ paraquat and survived longer than wild-type flies, whereas flies carrying mutations in ple or $\mathrm{Pu}$ were more sensitive to paraquat. This indicated that sensitivity to the environmental neurotoxin paraquat might be modified by variations in genes that regulate dopamine synthesis and metabolism.

These findings clearly show how genetic and environmental factors can interact to cause neurodegeneration in a Drosophila model. The genes ple, $P u$ and Catsup are conserved in humans, and it is possible that they might act in concert to influence susceptibility to developing Parkinson's disease after exposure to the herbicide. Leonie Welberg

ORIGINAL RESEARCH PAPER Chaudhuri, A. et a Interaction of genetic and environmental factors in a Drosophila Parkinsonism model.J. Neurosci. 27, 2457-2467 (2007) 